\title{
GEOMATICS MAPPING OF NATURAL HAZARDS: OVERVIEW AND EXPERIENCES
}

\author{
I. Toschi ${ }^{\text {a }}$, M. Allocca ${ }^{\text {a }}$, F. Remondino ${ }^{\text {a }}$ \\ a 3D Optical Metrology (3DOM) unit, Bruno Kessler Foundation (FBK), Trento, Italy - (toschi, allocca, remondino)@ fbk.eu
}

Commission III, ICWG III/IVa

KEY WORDS : Rapid Mapping, Multi-temporal, Geomatics, Platform, Sensor, Landslide, Mudflow, Time management

\begin{abstract}
:
This paper reviews the major Geomatics solutions available on the market and explores their potentialities for rapid disaster assessment applications. The attention is primarily focused on the most recent satellite and airborne / UAV optical imaging systems, with the goal of providing an update that will assist the selection process when involved in Rapid Mapping. Furthermore, two relevant and on-going experiences, carried out by the authors within international cooperation frameworks, are described with special focus on hazardous events or situations in the Alpine region: (i) Rapid Mapping of mudflow event, by exploiting LiDAR data and satellite, airborne, helicopter- and UAV-based imagery; (ii) multi-temporal monitoring for displacement assessment of landslide event, by integrating multi-sensor and multi-platform Geomatics techniques. Results and lessons learnt are presented that may support the choice of the most suited Geomatics technique.
\end{abstract}

\section{INTRODUCTION}

In case of natural hazards, a rapid assessment of the crisis situation is paramount to gather information on the magnitude of the disaster and the extent of its impact on the population, the infrastructures and the environment. In this regard, data remotely acquired from satellite, aircraft and UAV (unmanned aerial vehicle) platforms can be efficiently adopted within an immediate post-event phase, usually referred to as "Rapid Mapping", in order to support the spatial understanding of the phenomenon and the collection of standardized information. In particular, irrespective of the hazards type, Geomatics sensors and techniques can be efficiently adopted to collect the most significant information or data (Table 1, top), in accordance with standardization criteria and constraints (Boccardo, 2016; Table 1, bottom). A consistent effort should be thus devoted to the processing phase, in order to guarantee both the required metric quality (i.e. resolution and accuracy) and delivery time.

\begin{tabular}{|l|}
\hline \multicolumn{1}{|c|}{ Relevant information and data } \\
\hline - location and size of the impacted area; \\
- intensity of the damages; \\
zonation (i.e. different damage intensities within the \\
impacted area); \\
- location of victims; \\
\hline location of other areas potentially endangered. \\
\hline \multicolumn{1}{c|}{ Criteria and constraints } \\
- processed data should meet the required metric quality \\
- value-added information should be extracted from the \\
processed data, to support critical analy ses and decision \\
making; \\
both processed data and value-added information should be \\
delivered in a standard and documented format.
\end{tabular}

Table 1. Relevant information to be collected and criteria/constraints to be fulfilled.

Within the Geomatics domain, there is a multiplicity of platforms, sensors and techniques that could be used for data acquisition to support the main phases of the "Disaster Management Cycle" (UN-SPIDER, UNEP 2012). A number of review papers have been published in this field in the past decade, focusing on the use of (i) volunteered geographic information, in the preparedness and mitigation phases (Klonner et al., 2016); (ii) geophy sical techniques, in the risk assessment phase (Malehmir et al., 2016); (iii) satellite remote sensing sensors and data, in the mapping and monitoring phases (Voigt et al., 2016; Joyce et al., 2014; Joyce et al., 2009); (iv) UAVbased photogrammetry, in the risk monitoring and disaster management phases (Erdelj et al., 2017; Giordan et al., 2017; Petrides et al., 2017; Gomez and Purdie, 2016); and (v) remote sensing techniques, for the management of specific disasters' types (Williams et al., 2018; Casagli et al., 2017; Hoque et al. 2017; Molinari et al., 2017).

Following the overview presented in (Toschi et al., 2017), this paper concentrates on the Rapid Mapping phase immediately after a disaster and reviews the major geomatic solutions available in the market for rapid disaster assessment applications. The attention is primarily focused on both satellite and airborne optical imaging systems (platforms and sensors), since they offer a significant advantage compared to groundbased survey, namely the ability to cover large areas within a short time and with limited site access constraints. The goal is to explores their potentialities (along with their limits) and assist crisis managers, civil protection and the other involved actors while selecting the most appropriate sensor and survey technique. After a survey of the latest satellite, airborne and UAV-based solutions (Section 2), two relevant and on-going experiences carried out by the authors within the framework of international cooperation, are described in Section 3, with special focus on recent hazardous events or situations. First results allow to discuss some useful guidelines, especially in terms of (i) time management and data processing efficiency, and (ii) comparison and integration of data remotely captured from multiple sensors and platforms. General remarks and lessons learnt on the main advantages and disadvantages associated with each Geomatics solution are finally summarized in Section 4.

\section{GEOMATICS RAPID MAPPING SYSTEMS}

\subsection{Satellite optical solutions}

Earth observation (EO) satellites still represent the most adopted solution for crisis mapping and damage assessment, since they can provide for a regular and wide-ranging update on the status of hazards at several scales, from global, through regional, to national level. The latest state-of-the art solutions enable, in principle, the continuous monitoring of earth's surface changes, 
and feature improved systems parameters, including spatial, spectral and temporal resolution. Indeed, spaceborne remote sensing technology has been experiencing significant developments in recent decades, providing for an increasing availability of high-quality optical satellite images and, in some cases, a multiple daily coverage. In addition, new multi-sensor solutions have been introduced, that record different parts of the electromagnetic spectrum and allow multiple data to be acquired within a short time period. Furthermore, most of these data are either freely available or provided at relatively low cost through international open-access infrastructures. These include, among the others, the International Charter on Space and Major Disasters (The International Disaster Charter) at international level, and the Copernicus Emergency Management Service (Copernicus EMS) at European level. These initiatives, together with other national and international operating systems (www.un-spider.org/space-application/emergency-

mechanisms), offer open-access platforms and information services, delivering EO data for rapid surveying of the location, scale and severity of the disaster impact.

From a technological point of view, the bus and the payload are the two main components which constitute a spacecraft. While the former serves as platform for the payload and regulates the system parameters (i.e. attitude, electric power, temperature, communication and orbit), the payload is the sensing devise (incl. either one sensor or a group of sensors) that acquires the EO measurements. Focusing on satellite digital camera systems, the core sensing element still remains the linear CCD and the most popular imaging configurations are pushbroom, whiskbroom and frame systems (Abdullah, 2013). Among them, the pushbroom sensor type is the most adopted camera concept for image formation, both in its basic form (i.e. a single chip of pixels that form the pushbroom framelet) and in its variations (i.e. more than one framelet in the focal plane or discontinuous flamelet). In terms of imaging configurations, state-of-the-art spacecraft can acquire imagery in various modes (Toschi et al., 2017), providing for both the observation of the most critical areas (hot spots) and the tracking of irregular coastlines and paths. These improved system capabilities, along with an increasing investment in the spaceborne sector, encourage the use of EO satellite data for Rapid Mapping applications. Indeed, spaceborne remote sensing provides several advantages, i.e. (i) accessibility to remote and hazardous areas without risk; (ii) broad area coverage at relatively low cost; (iii) collection of consistent, standardized and comparable information on multiple scales. However, satellite images still suffer from a limited spatial resolution (usually, from $50 \mathrm{~cm}$ to few $\mathrm{m}$ ), if compared to airborne solutions; furthermore, optical satellite data are weather dependent, with limited usability in case of a significant cloudy coverage.

\begin{tabular}{|c|c|c|c|c|c|c|c|}
\hline & Mission/Sensor & Spectral bands & GSD $^{(*)}[\mathrm{m}]$ & Swath width $[\mathrm{km}]$ & Revisit time & Inclination $\left[{ }^{\circ}\right]$ & Altitude [km] \\
\hline \multirow{6}{*}{ 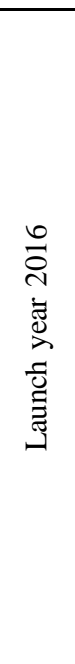 } & $\begin{array}{cc} & \text { PRISM- } \\
\text { ALOS-3/ } & 2 \\
& \text { HISUI }\end{array}$ & $\begin{array}{c}\text { Panchromatic } \\
\text { Multi spectral (4) } \\
\text { Hyper spectral }\end{array}$ & $\begin{array}{c}0.8 \\
5 \\
30\end{array}$ & $\begin{array}{l}50 \\
90 \\
30\end{array}$ & - & 97.9 & 618 \\
\hline & Diwata-1/HPT & RGB, NIR & 3 & \begin{tabular}{|c|} 
FOV of $1.9 \mathrm{~km} \times 1.4$ \\
$\mathrm{~km}$
\end{tabular} & - & 51.6 & 400 \\
\hline & FormoSat-5/RSI & $\begin{array}{c}\text { Panchromatic } \\
\text { Multi spectral (4) }\end{array}$ & $\begin{array}{l}2 \\
4\end{array}$ & 24 & $\begin{array}{l}\text { Every other } \\
\text { day }\end{array}$ & 98.28 & 720 \\
\hline & SuperView 1 & $\begin{array}{c}\text { Panchromatic } \\
\text { Multi spectral (4) }\end{array}$ & $\begin{array}{c}0.5 \\
2\end{array}$ & 12 & 4 day s & 98 & 530 \\
\hline & Göktürk-1/HiRI & $\begin{array}{c}\text { Panchromatic } \\
\text { Multi spectral (4) }\end{array}$ & $\begin{array}{l}0.7 \\
2.8\end{array}$ & 20 & $<2$ days & 98.11 & 695 \\
\hline & $\begin{array}{c}\text { WorldView- } \\
\text { 4/SpaceView }{ }^{\mathrm{TM}} 110\end{array}$ & $\begin{array}{c}\text { Panchromatic } \\
\text { Multi spectral (4) }\end{array}$ & $\begin{array}{l}0.31 \\
1.24\end{array}$ & 13.1 & $\leq 3$ days & 98 & 617 \\
\hline \multirow{5}{*}{ 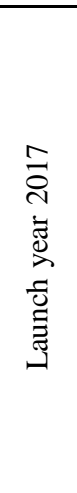 } & $\begin{array}{cc}\text { CartoSAT- } & \text { PAN } \\
\text { 2D/ } & \text { HRMX }\end{array}$ & $\begin{array}{c}\text { Panchromatic } \\
\text { Multi spectral (4) }\end{array}$ & $\begin{array}{c}0.65 \\
2\end{array}$ & $\begin{array}{l}9.6 \\
10\end{array}$ & 4 days & 97.44 & 505 \\
\hline & \begin{tabular}{|cc} 
Kanopus-V & PSS \\
& MSS
\end{tabular} & $\begin{array}{c}\text { Panchromatic } \\
\text { Multi spectral (4) }\end{array}$ & $\begin{array}{l}2.5 \\
12\end{array}$ & 20 & - & - & 510 \\
\hline & KhalifaSat/ KHCS & $\begin{array}{c}\text { Panchromatic } \\
\text { Multi spectral (4) } \\
\end{array}$ & $\begin{array}{l}1 \\
4 \\
\end{array}$ & 12 & - & 98.13 & 613 \\
\hline & $\begin{array}{c}\text { Sentinel-2B (twin } \\
\text { satellite Sentinel- } \\
\text { 2A)/MSI } \\
\end{array}$ & Multi spectral & $\begin{array}{c}10 \mathrm{~m} \\
\text { (4 bands) }\end{array}$ & 290 & \begin{tabular}{|c|}
5 days (full \\
mission of \\
twin satellites) \\
\end{tabular} & 98.62 & 786 \\
\hline & VEN $\mu \mathrm{S} / \mathrm{VSSC}$ & Super spectral (12) & 5.3 & 27.56 & 2 days & 98.28 & 720 \\
\hline 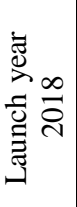 & PRISM A/PRISMA & Hyperspectral & $\begin{array}{c}5 \text { (Pan) } \\
30 \text { (VNIR) } \\
30 \text { (SWIR) }\end{array}$ & 30 & 7 days & 97.85 & 615 \\
\hline
\end{tabular}

Table 2. Satellite solutions: latest missions with optical sensors and high spatial resolution. ${ }^{(*)}$ Ground Sample Distance at nadir. 
Table 2 lists the most recent (from 2016 up to now) optical satellite solutions, with special focus on high and very highresolution sensors (Ground Sample Distance, GSD, below 10 m). A more comprehensive overview of EO satellites can be found in the online databases provided by the European Space Agency (eoPortal) and the Committee on Earth Observation Satellites (CEOS Database)

\subsection{Airborne and UAV optical solutions}

When high resolution imagery (from few $\mathrm{cm}$ to few $\mathrm{mm}$ ), close range to target and high flexibility are needed, airborne solutions are generally preferred thanks to their efficiency of data acquisition. The airborne remote sensing industry is experiencing an unpreceded diversity in the development of platforms and sensors. Therefore, Rapid M apping surveys after disaster events can now be performed using a number of different airborne Geomatics solutions.

Many types of airborne platforms exist, that enable optical sensors to reach and maintain the desired operating conditions (altitude, viewing angle, etc.). These include:

- aircraft platforms, that can provide for high spatial resolution imagery (up to few $\mathrm{cm}$ ) and high efficiency of data acquisition (i.e. larger area coverage per unit of time). However, few limitations should be considered when opting for manned aircrafts, such as constraints in the flight management (minimal flight speed and altitude, no curves and corridors, no operations allowed during hazardous weather conditions), more efforts in the flight planning (rigorous flight plan), and limited flight duration due to crew duties. In terms of platform type, twin-engine aircrafts still represent the most adopted solution for data collection, thanks to their broad area coverage capabilities and higher efficiency, driven by gains in aircraft airspeed and power. Their sensor complement usually consists of an integrated multi-sensor system (incl. cameras, LiDAR, navigation and positioning systems, data storage and management units, etc.). On the other hand, the advent of high-quality small-format digital cameras is now pushing the use of lighter single-engine aircrafts, that enable slow, low-altitude and single-person operations with modest payloads.

- helicopter platforms, that represent an efficient mapping means for quick response applications thanks to their close-range to target scenes, good maneuverability (even in case of bad weather conditions), freedom to operate, and easily access in congested airspace. Helicopters are particularly preferred over fixed-wing aircrafts when flight characteristics require low altitude, low speed or following curves/corridor trajectories. In terms of sensors' configuration, they can feature both internal and external sensor mounting setup, along with the adoption of handheld acquisition systems.

- UAV platforms, that offer high flexibility and maneuverability, very high image resolution (up to few $\mathrm{mm}$ ), adaptability to fly at different altitudes and ability to access remote and dangerous environments without any risk for the pilot. They come nowadays in many different forms, usually categorized by size, weight, flying altitude, payload and endurance. Their increasing use for remote sensing applications (Gomez and Purdie, 2016; Colomina and Molina, 2014; Nex and Remondino, 2014) is also driven by the rapid development of UAV technologies and the miniaturization of on-board equipment, that provide the user with several solutions. Key to the selection of the most appropriate one is the type of target scene: while multi-rotor platforms represent the best mapping choice for small and isolated areas or buildings, when it comes to the survey of large areas, fixed-wing platforms speed up the acquisition time and are usually preferred. If compared to other airborne platforms, UAVs show disadvantages concerning endurance, payload and area coverage limitations.

With regards to the payload component, the evolution in digital technology has provided for a suite of remote sensing sensors that can be efficiently used for Rapid Mapping applications These include:

- airborne digital camera systems, usually classified as small, medium and large format digital cameras. Among them, medium format digital cameras (Table 3), featuring an image resolution in the range $30-100 \mathrm{Mpx}$, currently represent the fastest growing segment of the airborne mapping market, since they offer the best flexibility in terms of data acquisition. Their use is usually oriented to support applications where moderate to high resolution is required and/or to integrate multi-sensors airborne platforms, as companion sensors for LiDAR systems.

- $\quad$ airborne digital multi-camera systems (often called oblique systems), consisting of a combination of two cameras (fan configuration), five cameras (Maltese-cross configuration), or, more recently, an even greater number of cameras (block configuration). Hybrid configuration, including a LiDAR unit, is also possible (e.g. CityMapper, by Leica Geosystems). The use of oblique systems in Rapid Mapping applications is driven by their efficiency in data acquisition and capacity to image building façades and footprints. However, they still represent a bulky, heavy and costly solution and require a proper flight planning to be carried out in order to cope with efficiency issues during the post-processing phase.

- small and medium format digital camera solutions for UAV platforms, usually categorized based on their spectral resolution. Among them, the sector of RGB cameras (Table 4) is rather mature to offer high-quality, highresolution and low-cost solutions, suitable for micro, min and tactical UAV payloads. Besides these, several multispectral, hyperspectral and thermal camera solutions exist (e.g. Micro-MCA6 and Macaw, by Tetracam; RedEdge and Parrot Sequoia, by MicaSense; Hyperspectral Camera, by Rikola; Tau 2 640, by FLIR), that could also be efficiently used for hazard mapping.

- LiDAR (Light Detection And Ranging) airborne systems, as stand-alone active sensor component or in combination with medium-format digital cameras. LiDAR is widely used to generate high-quality, very high resolution DEMs (Digital Elevation Models) for terrain analysis, to map landslides and debris-flows, to monitor mass movements, and to refine surface flow models. Furthermore, if combined with imagery, it offers several advantages, such as direct digital terrain/surface model (DTM/DSM) generation, DTM/DSM integration with RGB information and orthophoto generation. Besides the traditional use of LiDAR technology in airborne photogrammetry applications, a few recent solutions exist, that integrate LiDAR unit as sensor component of UAV platforms (e.g. Mapper II, by YellowScan; VLP-16, by Velodyne; VUXIUAV, by Riegl). Although these systems show promising results, the transfer of LiDAR to UAV is still challenging, either due to the difficult trade-off between performance and size or cost of LiDAR, or due to the effect of flight dynamics on the measurement process. Furthermore, to our knowledge, in case of Rapid Mapping situation, mainly optical imaging sensors are used. 


\begin{tabular}{|c|c|c|c|c|c|}
\hline Model & Manufacturer & $\begin{array}{c}\text { Geometric } \\
\text { resolution [MPx] }\end{array}$ & Pixel size $[\mu \mathrm{m}]$ & $\begin{array}{c}\text { Sensor type } \\
\text { and no. of frames }{ }^{(*)} / \text { lines }\end{array}$ & Spectral bands \\
\hline $\begin{array}{c}\text { Wehrli/Geosystem } \\
\text { pushbroom sy stems: } \\
\text { 3-DAS-1 } \\
\text { 4-DAS-1 }\end{array}$ & $\begin{array}{c}\text { Wehrli/ } \\
\text { Geosystem }\end{array}$ & 8002 px per line & 9 & $\begin{array}{l}\text { Linear (3) } \\
\text { Linear (4) }\end{array}$ & $\begin{array}{c}\text { RGB } \\
\text { RGB and NIR }\end{array}$ \\
\hline $\begin{array}{c}\text { Trimble Aerial Cameras: } \\
\text { P45+ } \\
\text { P65+ } \\
\text { IQ180 }\end{array}$ & Trimble & $\begin{array}{l}39 \\
60 \\
80\end{array}$ & $\begin{array}{c}6.8 \\
6 \\
5.2\end{array}$ & $\begin{array}{l}\text { Frame } \\
\text { Frame } \\
\text { Frame }\end{array}$ & $\begin{array}{l}\text { RGB } \\
\text { RGB } \\
\text { RGB }\end{array}$ \\
\hline CS- 10000 & Optech & 80 & 5.2 & Frame & RGB \\
\hline $\begin{array}{c}\text { DigiCAM: } \\
\text { DigiCAM } \\
\text { Dual DigiCAM }\end{array}$ & IGI & $\begin{array}{c}40,50,60,80 \\
76,98,118\end{array}$ & $\begin{array}{l}6,6,6,5.2 \\
6.8,6,6\end{array}$ & $\begin{array}{l}\text { Frame } \\
\text { Frame }\end{array}$ & $\begin{array}{l}\text { RGB or CIR } \\
\text { RGB or CIR }\end{array}$ \\
\hline Leica-RCD100 & $\begin{array}{c}\text { Leica } \\
\text { Geosystems }\end{array}$ & 39 & 6.8 & $\begin{array}{c}\text { Frame }(1 \text { or } 2 \mathrm{CH} 39 \text { camera } \\
\text { heads })\end{array}$ & RGB or CIR \\
\hline RMK D Camera System & Intergraph & 37 & 7.2 & Frame (4 camera heads) & RGB, NIR \\
\hline UltraCam-Lp system & Vexcel Imaging & $\begin{array}{l}92 \text { (PAN, } 2 \text { cones), } \\
20 \text { (MS, } 2 \text { cones) }\end{array}$ & 6 & Frame (4) & PAN, RGB, NIR \\
\hline
\end{tabular}

Table 3. Airborne solutions: most common medium format airborne digital cameras. ${ }^{(*)}$ If the system includes more than one frame.

\begin{tabular}{|c|c|c|c|c|c|}
\hline Model & Manufacturer & $\begin{array}{c}\text { Geometric } \\
\text { resolution }[\mathrm{MPx}]\end{array}$ & Pixel size $[\mu \mathrm{m}]$ & Weight $[\mathrm{kg}]$ & Notes \\
\hline albris $^{(*)}$ & senseFly & 38 & 1.4 & - & - \\
\hline $\begin{array}{l}\text { iXA } 160 \\
\text { iXA } 180\end{array}$ & Phase One & $\begin{array}{c}60.5 \\
80\end{array}$ & $\begin{array}{c}6 \\
5.2\end{array}$ & 1.75 & CCD sensor \\
\hline $\begin{array}{c}\text { P65+ } \\
\text { IQ180 }\end{array}$ & Trimble & $\begin{array}{l}60 \\
80\end{array}$ & $\begin{array}{c}6 \\
5.2\end{array}$ & 1.50 & CCD sensor \\
\hline H5D-60 & Hasselblad & 60 & 6 & 2.29 (incl. lens) & CCD sensor \\
\hline Phantom 4 Pro ${ }^{(*)}$ & DJI & 20 & 2.3 & - & CMOS sensor \\
\hline Sony Alpha NEX-7 & Sony & 24.3 & 3.9 & 0.4 & CMOS sensor \\
\hline Sony Alpha NEX-5N & Sony & 16 & 4.7 & 0.27 & CMOS sensor \\
\hline Sony Alpha a6000 & Sony & 24 & 3.9 & 0.34 & CMOS sensor \\
\hline GXR A16 & $\mathrm{RICOH}$ & 16.2 & 4.8 & 0.35 & CMOS sensor \\
\hline $\begin{array}{c}\text { Zenmuse Gimbal } \\
\text { Cameras }^{(*)} \text { : } \\
\text { Zenmuse X7 } \\
\text { Zenmuse X5S } \\
\text { Zenmuse X4S }\end{array}$ & DJI & $\begin{array}{c}24 \\
20.8 \\
20\end{array}$ & $\begin{array}{l}3.9 \\
3.2 \\
2.3\end{array}$ & $\begin{array}{l}0.45 \\
0.46 \\
0.25\end{array}$ & CMOS sensor \\
\hline
\end{tabular}

Table 4. UAV solutions: most common small and medium format digital cameras for UAVs. ${ }^{(*)}$ Solutions with integrated camera.

\section{EXPERIENCES}

\subsection{Rapid Mapping of mudflow events}

A series of mudflow events, triggered by a rockfall of over 3 Mio $\mathrm{m}^{3}$, reached the Bregaglia valley, near the Swiss-Italian border, in August 2017. The little village of Bondo (Switzerland), was particularly devastated by the events, that caused victims and significant damages to buildings and infrastructures. In the immediate post-disaster phase, a multisensor and multi-platform data acquisition campaign was conducted by the Federal Office of Topography swisstopo, including the collection of high resolution, multispectral WorldView-4 satellite imagery, aerial imagery with ADS100 camera, helicopter-based optical imagery and LiDAR data, as well as UAV-based images. These data, together with predisaster mapping products, represent a unique dataset to perform a benchmark analysis of different sensors and processing workflows with respect to Rapid Mapping. With this in mind, a joint research project was initiated to test the applicability of the different sensors, along with the corresponding data processing workflows over the hazard area. In particular, comparative tests are currently carried out to (i) highlight the pros and cons in terms of platform, sensor, and production pipeline; (ii) evaluate the performance of the processing workflows, especially with respect to their time efficiency; (iii) give suggestions to optimise the existing Rapid Mapping services.

With respect to time management in a photogrammetric project, several solutions are evaluated and compared to achieve the best performance in both data acquisition and processing. The latter, in particular, should be carefully planned in order to provide for the highest post-processing efficiency, that is usually defined as the time required to process a given number of images. Obviously, this first depends on the available computing capacities (i.e. hardware and software). Second, the number of 
images and their geometric and radiometric resolution, represent key-factors to define the dimension of a project. However, the most important issue affecting the photogrammetric postprocessing time is still the strategy adopted to retrieve the camera exterior orientation parameters. Advancements in Global Navigation Satellite System (GNSS) hardware and software have triggered the development of alternative methods to traditional aerial triangulation (AT), that was originally dependent on a sufficient number of well distributed ground control points (GCP). If the use of GNSS-derived camera position data within the bundle block adjustment (i.e. GNSSsupported aerial triangulation) allows to save time and cost by reducing the need for ground control, the highest gain would be achieved by skipping the triangulation of the image block through direct georeferencing (DG). Obviously, the applicability of this approach depends on the accuracy of the available GNSS/IMU data and on the quality requirements of the final Rapid Mapping products.

The initial tests are performed using the UAV dataset which includes 163 images acquired with the multi-rotor albris by senseFly. The platform is equipped with multiple sensors, comprising a RGB camera ( $8 \mathrm{~mm}$ focal length, $1.4 \mu \mathrm{m}$ pixel size), a thermal and a video camera. Two flights were performed at a mean altitude of $100 \mathrm{~m}$ over the Bondo area (ca. $500 \times 400 \mathrm{~m}$ ), resulting in a mean GSD of $1.8 \mathrm{~cm}$. Ground control is provided in the form of 10 points, whose planimetric position was derived from SWISSIMAGE orthophoto $(0.25 \mathrm{~m}$ spatial resolution) whereas the height was measured in swissALTI3D elevation model $(1 \sigma=0.5-3 \mathrm{~m})$. Several strategies for feature-based matching and image orientation have been tested in Pix4Dmapper (Pix4D), including:

- tie point extraction at different image pyramid levels (i.e image scale $1,1 / 2$ and $1 / 4$ );

- $\quad$ direct georeferencing (DG), by directly deriving exterior orientation parameters from on-board GNSS/IMU data;

- GNSS-supported aerial triangulation;

- GNSS- and GCP-supported aerial triangulation;

- Free-network adjustment (no GCPs, no GNSS observations), followed by a rigid similarity transformation (Helmert trasformation) on well distributed GCPs.

First results are listed in Table 5 (comparison of time values) and Table 6 (comparison of accuracy values). Accuracy is expressed as root mean square error (RMSE) on 5 check points (CPs). The spatial distribution of control points is shown in Figure 1, whereas Figure 2 reports two details of the generated dense point clouds over the hazard area (mean spatial resolution of $2 \mathrm{~cm}$ ). Finally, Figure 3 displays a shaded slope map derived from the photogrammetric DSM (spatial resolution of 2xGSD).

\begin{tabular}{|c|c|c|c|}
\cline { 2 - 4 } \multicolumn{1}{c|}{} & \multicolumn{3}{c|}{ Image scale } \\
\cline { 2 - 4 } \multicolumn{1}{c|}{} & 1 (full res.) & $1 / 2$ & $1 / 4$ \\
\hline DG $^{(*)}$ & $13 \mathrm{~min} 10 \mathrm{sec}$ & $7 \mathrm{~min} 34 \mathrm{sec}$ & $2 \mathrm{~min} 44 \mathrm{sec}$ \\
\hline $\begin{array}{c}\text { GNSS-supported } \\
\text { AT }\end{array}$ & $32 \mathrm{~min} 34 \mathrm{sec}$ & $21 \mathrm{~min} 08 \mathrm{sec}$ & $7 \mathrm{~min} 40 \mathrm{sec}$ \\
\hline $\begin{array}{c}\text { GNSS- and GCP- } \\
\text { supported AT }\end{array}{ }^{(*)}$ & $36 \mathrm{~min} 43 \mathrm{sec}$ & $22 \mathrm{~min} 8 \mathrm{sec}$ & $8 \mathrm{~min} 36 \mathrm{sec}$ \\
\hline $\begin{array}{c}\text { Free-Net AT }+ \\
\text { Helmert }\end{array}$ & $22 \mathrm{~min} 06 \mathrm{sec}$ & $14 \mathrm{~min} 32 \mathrm{sec}$ & $6 \mathrm{~min} 35 \mathrm{sec}$ \\
\hline
\end{tabular}

Table 5. Comparison of processing time values. ${ }^{(*)}$ Times are referred to the tie point extraction task, that is a mandatory step of the Pix4D workflow. ${ }^{(* *)}$ Time for control points 2D/3D measurement is not included. The longer processing time is due to the optimization of the initial bundle results exploiting the GCPs observations.

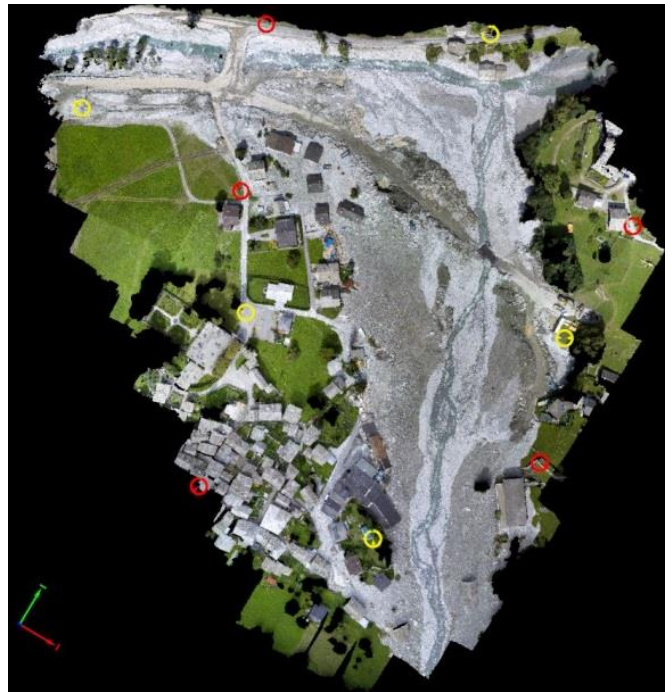

Figure 1. Distribution of 5 GCPs (yellow points) and 5 CPs (red points) on the dense point cloud.
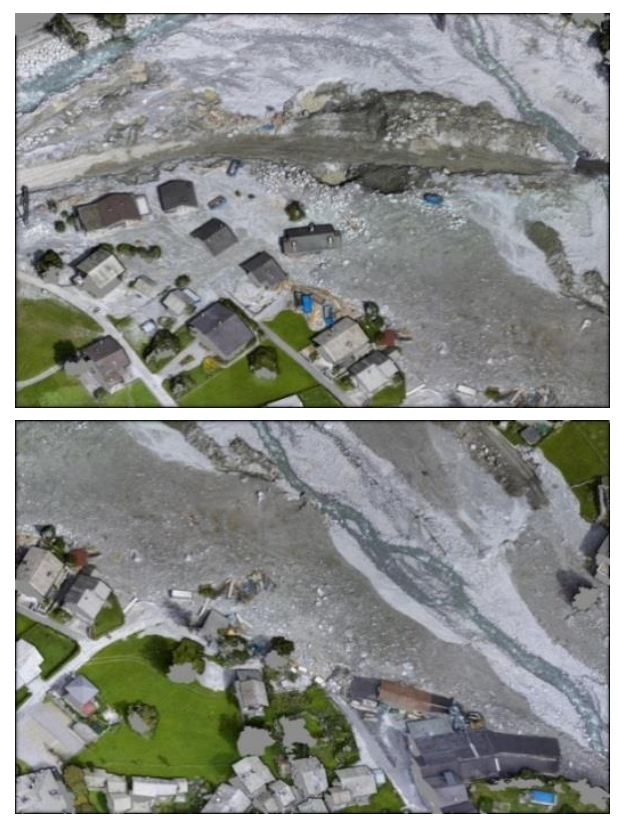

Figure 2. Close-up views of the generated dense point cloud.

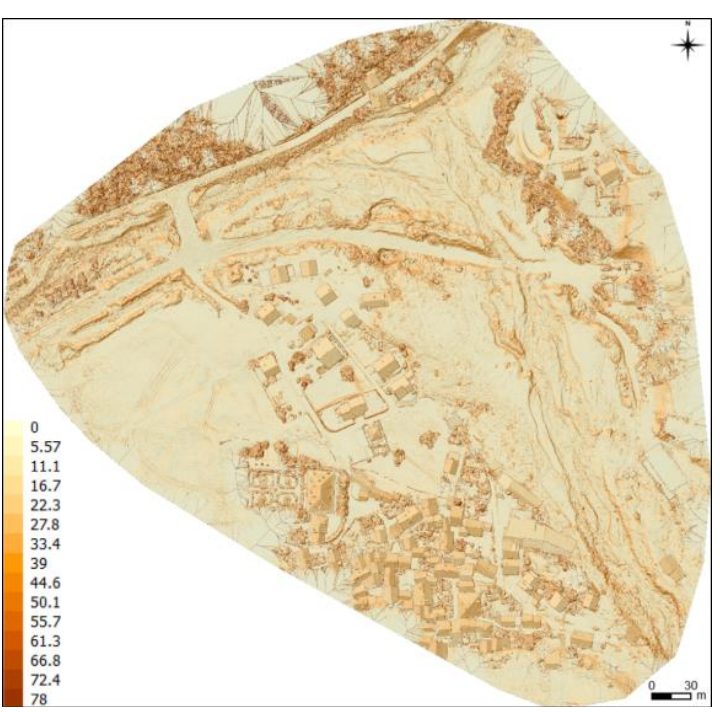

Figure 3. Shaded maps of slopes (in percentage). 


\begin{tabular}{|c|c|c|c|c|c|c|c|c|c|}
\cline { 2 - 10 } \multicolumn{1}{c|}{} & \multicolumn{3}{c|}{ Image scale 1 (full res.) } & \multicolumn{3}{c|}{ Image scale 1/2 } & \multicolumn{3}{c|}{ Image scale 1/4 } \\
\cline { 2 - 10 } \multicolumn{1}{c|}{} & $\mathrm{X}$ & $\mathrm{Y}$ & $\mathrm{Z}$ & $\mathrm{X}$ & $\mathrm{Y}$ & $\mathrm{Z}$ & $\mathrm{X}$ & $\mathrm{Y}$ & $\mathrm{Z}$ \\
\hline $\mathrm{DG}^{(*)}$ & 2.453 & 5.373 & 13.223 & 2.413 & 5.417 & 12.321 & 1.984 & 6.758 & 19.561 \\
\hline GNSS-supported & 1.884 & 7.481 & 10.864 & 1.876 & 7.489 & 10.851 & 1.869 & 7.548 & 10.579 \\
\hline $\begin{array}{c}\text { GNSS- and GCP- } \\
\text { supported AT }\end{array}$ & 0.165 & 0.111 & 1.007 & 0.163 & 0.107 & 1.002 & 0.173 & 0.101 & 1.016 \\
\hline $\begin{array}{c}\text { Free-network AT } \\
\text { + Helmert }\end{array}$ & 0.122 & 0.109 & 0.260 & 0.129 & 0.090 & 0.277 & 0.143 & 0.093 & 0.288 \\
\hline
\end{tabular}

Table 6. Comparison of accuracy values, computed as RMSE (X, Y, Z) on 5 check points [m]. ${ }^{(*)}$ Errors on forward-intersected points vary with the image scale, because some images are not oriented and automatically disabled by Pix4D.

From this hazard, the following remarks can be summarized:

- a suitable choice of the image scale adopted for featurebased matching is a key-factor to reduce the processing time;

- the adoption of full image resolution doesn't provide for a significant increase in object-space accuracy, if compared to requirements needed for emergency mapping purposes;

- $\quad$ DG and GNSS-assisted AT (i.e. those solutions that do not require for control points to be measured) can provide for an average horizontal accuracy of about $4 \mathrm{~m}$, that may support the needs of the immediate response phase;

- the use of GCPs derived from pre-existing mapping products is influenced by their limited quality. To avoid this decrease in AT accuracy (particularly, along Z), a rigid similarity transformation could be preferred over a classical GNSS-assisted AT to improve accuracy.

\subsection{Displacement assessment of landslide events}

Landslide monitoring is paramount to assess landslide activity and prevent potential impacts on people and buildings. For this task, various monitoring techniques have been proposed, suitable for mapping the active areas at subsequent epochs and quantifying landslide displacements at characteristic points, along profiles or area-wide. Particularly, terrestrial and airborne 3D imaging sensors can provide area-wide information about surface characteristics and its changes over time. However, each sensor, and corresponding measurement principles, features specific characteristics such as spatial, temporal, spectral and radiometric resolution, that make it more or less eligible for a defined monitoring task. A combination of different platforms and sensors may thus overcome the limitations of individual devices, by extending their specific spatial/temporal coverage and lowering the global uncertainty.

With this in mind, an international consortium of remote sensing and mapping experts was built, under the framework of the Euregio funding scheme, to integrate their expertise within the LEMONADE project (landslide monitoring and data integration - http://lemonade.mountainresearch.at). This ongoing project aims to develop a multi-sensor and multi-temporal data fusion approach for the area-wide assessment of landslide displacements. Since main efforts are focused on investigating advantages and limitations of different mapping techniques, this will serve as support to the decision-making and selection process when involved in hazard mapping and monitoring exercises. As real-case scenarios, three landslide sites in the Alps were identified: Schmirn (Tyrol, Austria), Fortebuso (Trento, Italy), and Corvara (South Tyrol, Italy). In particular, the latter represents a suitable test site, where different remote sensing techniques can be efficiently adopted, namely (i) InSAR (interferometric synthetic aperture radar); (ii) terrestrial and UAV-based photogrammetry and (iii) terrestrial laser scanning (Rutzinger et al., 2017; Schlögel et al., 2017; Thiebes et al., 2016).

Focusing on the photogrammetric acquisition and processing, a RICOH GR compact camera $(18.3 \mathrm{~mm}$ focal length, $4.6 \mu \mathrm{m}$ pixel size), onboard the Octagon $\mathrm{x}-8$ platform, was adopted to survey the active part of the Corvara landslide (ca. $400 \times 250 \mathrm{~m}$ ) in August 2015 and June 2016. At the same time, two terrestrial photogrammetric campaigns were carried out on a sub-area (ca. $80 \times 70$ m, Figure 4, a), using a Nikon D750 digital camera (6 $\mu \mathrm{m}$ pixel size) equipped with a AF Nikkor $50 \mathrm{~mm} \mathrm{f} / 1.8 \mathrm{D}$ prime lens. Camera networks were carefully planned to achieve in both epochs a mean GSD of $1.8 \mathrm{~cm}$ and $0.5 \mathrm{~cm}$ for the UAV and terrestrial photogrammetric projects, respectively. Furthermore, a well-distributed set of GNSS-measured targets served as control points to define a common reference system and check the accuracy of image triangulation (average RMSE $\mathrm{XY} / \mathrm{Z}$ of $2.5 / 3.5 \mathrm{~cm}$ for the UAV network, $1.0 / 1.3 \mathrm{~cm}$ for the terrestrial network). After camera interior and exterior parameters were estimated, photogrammetric point clouds were generated based on the SURE workflow (nFrames) and further products derived. Figure $4 \mathrm{a}$ shows the UAV-based DSM cloud (1 GSD resolution), whereas two details of the dense point cloud retrieved by terrestrial photogrammetry (mean spatial resolution of $0.5 \mathrm{~cm}$ ) are displayed in Figure $4 \mathrm{~b}$. Furthermore, two value-added products are shown in Figure 5: the map of multi-temporal DSM elevation differences (Figure 5a) and the supervised land-cover classification map (Figure 5b), generated from the RGB orthophoto (Figure $5 \mathrm{c}$ ). The land-cover classification of the orthophoto is achieved by applying a sequential classification approach, including: (i) an initial unsupervised classification step, aimed at identifying the training samples; (ii) a supervised classification step, using the training samples extracted by the previous step. Within the LEMONADE project activities, this additional raster product is used as support for the multi-sensor integration task, that will consider the varying performance of each technique with the characteristics of the surveyed area.

For this hazard the following remarks can be summarized:

- UAV surveys allow a quick metric analy sis of large hazard areas with resolution and accuracy in the $\mathrm{cm}$ domain.

- elevation differences computed on multi-epoch photogrammetric DSM allow to analyse area-wide landslide dynamics. However they are affected by issues related to the measurement technique, e.g. occlusions due to vegetation, georeferencing uncertainty, noise in the dense image matching, etc. 


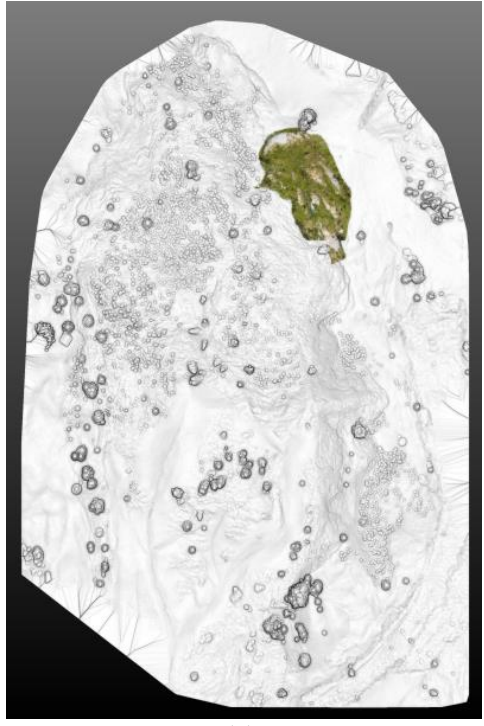

(a)

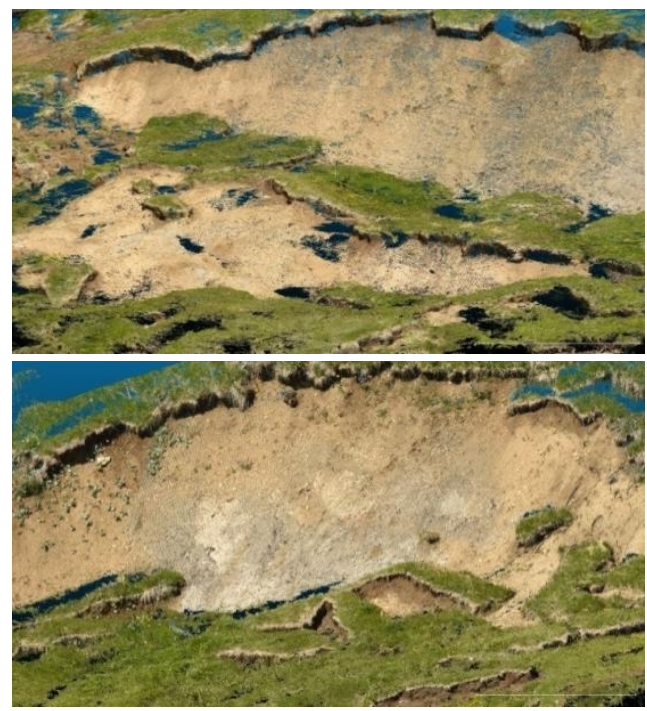

(b)

Figure 4. Two 3D products generated with photogrammetry over the landslide: (a) DSM cloud of the entire area, with a 3D dense colour cloud of the most active sub-area; (b) close-up views of the terrestrial dense point cloud.

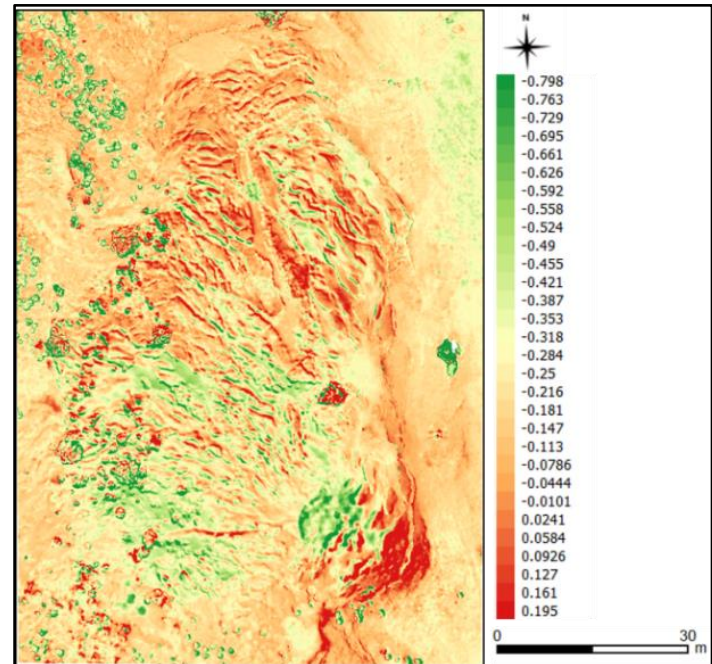

(a)

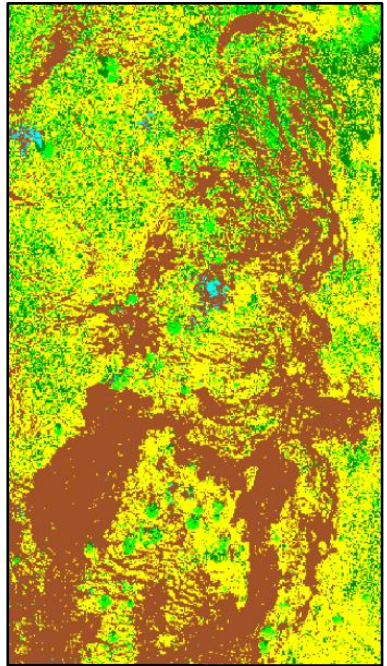

(b)

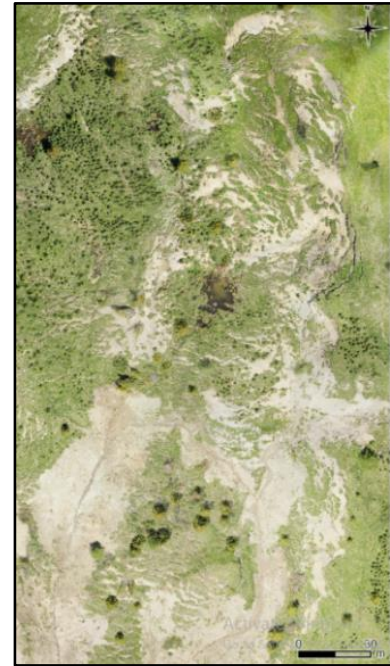

(c)

Figure 5. Three 2D products generated with photogrammetry: (a) DSM elevation differences $(2016$ - 2017) over the most active subarea; (b) supervised land-cover classification map (cyan: water, brown: bare soil, green: high vegetation, light green: mid vegetation, yellow: low vegetation); (c) RGB orthophoto at $2 \mathrm{~cm}$ resolution.

- although UAV blocks can reach cm-level GSD, the aforementioned issues could prevent an accurate assessment of slow displacements

- $\quad$ these limits will be compensated by integrating terrestrial laser scanning data, that offer few advantages in terms of vegetation penetration and high reliability.

\section{CONCLUS IONS}

There is a number of different Geomatics solutions for approaching a Rapid Mapping task. In many cases, the final decision on the data type and processing technique will depend on the equipment availability (platform and sensor), location and, sometimes, project funding. This paper provided a review of the current state-of-the-art remote sensing optical techniques (both satellite and airborne/UAV) for Rapid Mapping purposes. Furthermore, two on-going projects have been presented, in order to critically reflect on the usefulness and applicability of the different sensors and datasets in "real" case studies. Some conclusive remarks can be drawn as follows:

- although there is a multiplicity of platforms and remote sensing sensors that could be used for data acquisition, the unpredictability and variety of hazards do not usually allow for a single all-encompassing solution to be adopted. Therefore, the choice of the sensor and the survey technique often remains the most critical decision.

- the integration of data acquired by different sensors and platforms may compensate for issues affecting each dataset and extended the spatial and temporal coverage. However, this requires a proper error budget to be prepared in advance, in order to estimate the most significant uncertainty components affecting each technique.

- the choice of the most appropriate sensors (or, the combination thereof) is not enough, since valuable information should be delivered in a very short time span Thus an efficient time management is paramount to meet the project objectives at minimal cost in terms of timing. 


\section{ACKNOWLEDGEMENTS}

The authors would like to acknowledge the Federal Office of Topography swisstopo (T. Kellenberger and A. Streilein) for supporting the Swiss project and providing the datasets over Bondo, as well as Euregio for supporting the LEMONADE project and its partners (M. Rutzinger, T. Zieher, R. Schlögel, C. Kofler, M. Darvishi and A. Mejia Aguilar) for their contribution in data acquisition and processing.

\section{REFERENCES}

Abdullah, Q.A., 2013. Photogrammetric platforms. In: Manual of Photogrammetry, J. Chris McGlone (Ed.), ASPRS, Sixth Edition, pp. 1318.

Boccardo, P., 2016. Geomatics and Emergency Management. Workshop "Multidisciplinary and transnational applications of Geomatics", Congresso SIFET 2016.

Casagli, N., Frodella, W., Morelli, S., Tofani, V., Ciampalini, A., Intrieri, E., Raspini, F., Rossi, G., Tanteri, L. and Lu, P., 2017. Spaceborne, UAV and ground-based remote sensing techniques for landslide mapping, monitoring and early warning. In: Geoenvironmental Disasters, 4(1), p.9.

CEOS Database - http://database.eohandbook.com/ (Last accessed on 2018, Feb. 12).

Colomina, I. and Molina, P., 2014. Unmanned aerial systems for photogrammetry and remote sensing: A review. In: ISPRS Journal of Photogrammetry and Remote Sensing, 92, pp.79-97.

Copernicus EMS. Emergency Management Service emergency.copernicus.eu/mapping (Last accessed on 2018, Feb. 12).

eoPortal - https://directory.eoportal.org/web/eoportal/satellitemissions (Last accessed on 2018, Feb. 12).

Erdelj, M., Król, M. and Natalizio, E., 2017. Wireless Sensor Networks and Multi-UAV Systems for Natural Disaster M anagement. In: Computer Networks, 124, pp.72-86.

Giordan, D., Manconi, A., Remondino, F., Nex, F., 2017: Use of unmanned aerial vehicles in monitoring application and management of natural hazards. In: Geomatics, Natural Hazards and Risk, 8(1), pp. 1-4.

Gomez, C., and Purdie, H., 2016. UAV-based Photogrammetry and Geocomputing for Hazards and Disaster Risk MonitoringA Review. In: Geoenvironmental Disasters, 3(1), pp. 1-11.

Hoque, M.A.A., Phinn, S., Roelfsema, C. and Childs, I., 2017. Tropical cyclone disaster management using remote sensing and spatial analy sis: a review. In: International Journal of Disaster Risk Reduction, 22, pp. 345-354.

Joyce, K.E., Belliss, S.E., Samsonov, S.V., McNeill, S.J. and Glassey, P.J., 2009. A review of the status of satellite remote sensing and image processing techniques for mapping natural hazards and disasters. In: Progress in Physical Geography, 33(2), pp.183-207.

Joyce, K.E., Samsonov, S.V., Levick, S.R., Engelbrecht, J. and Belliss, S., 2014. Mapping and monitoring geological hazards using optical, LiDAR, and synthetic aperture RADAR image data. In: Natural hazards, 73(2), pp.137-163.

Klonner, C., Marx, S., Usón, T., Porto de Albuquerque, J. and Höfle, B., 2016. Volunteered geographic information in natura hazard analysis: a systematic literature review of current approaches with a focus on preparedness and mitigation. In: ISPRS International Journal of Geo-Information, 5(7), p.103.

Malehmir, A., Socco, L.V., Bastani, M., Krawczyk, C.M., Pfaffhuber, A.A., Miller, R.D., Maurer, H., Frauenfelder, R., Suto, K., Bazin, S., Merz, K., 2016. Near-Surface Geophy sical Characterization of Areas Prone to Natural Hazards: A Review of the Current and Perspective on the Future. In: Advances in Geophysics, 57, pp.51-146.

Molinari, D., Menoni, S. and Ballio, F., 2017. Flood Damage Survey and Assessment: New Insights from Research and Practice. John Wiley \& Sons, pp. 257.

Nex, F. and Remondino, F., 2014. UAV for 3D mapping applications: a review. In: Applied Geomatics, 6(1), pp.1-15.

nFrames, SURE - www.nframes.com Last accessed on 2018, Feb. 12).

Petrides, P., Kolios, P., Kyrkou, C., Theocharides, T. and Panayiotou, C., 2017. Disaster Prevention and Emergency Response Using Unmanned Aerial Systems. In: Smart Cities in the Mediterranean, pp. 379-403.

Pix4D, Pix4Dmapper - www.pix4d.com (Last accessed on 2018, Feb. 12).

Rutzinger, M., Zieher, T., Pfeiffer, J., Schlögel, R., Darvishi, M., Toschi, I. and Remondino, F., 2017. Evaluating synergy effects of combined close-range and remote sensing techniques for the monitoring of a deep-seated landslide (Schmirn, Austria). In: EGU Geophysical Abstracts, 19, EGU2017-63933.

Schlögel, R., Darvishi, M., Cuozzo, g., Kofler, c., Rutzinger, M., Zieher, T., Toschi, I. and Remondino, F., 2017. Sentinel-1 and ground-based sensors for a continuous monitoring of the Corvara landslide kinematic (South Tirol, Italy). In: EGU Geophysical Abstracts, 19, EGU2017-12913.

The International Disaster Charter - www.disasterscharter.org (Last accessed on 2018, Feb. 12)

Thiebes, B., Tomelleri, E., Aguilar, A., Schlögel, R., Darvishi, M., Remondino, F., Toschi, I., Rutzinger M., and Zieher, T., 2016. UAV-based landslide monitoring - first results from Corvara landslide, Italy. In: EGU Geophysical Abstracts, 18, EGU2016-12115.

Toschi, I., Remondino, F., Kellenberger, T. and Streilein, A., 2017. A Survey of Geomatics Solutions for the Rapid Mapping of Natural Hazards. In: Photogrammetric Engineering \& Remote Sensing, 83(12), pp.843-859.

UNEP - United Nations Environment Programme, 2012. Early Warning Systems: A State of the Art Analysis and Future Directions. Division of Early Warning and Assessment (DEWA), United Nations Environment Programme (UNEP).

UN-SPIDER- United Nations Platform "SPace-based Information for Disaster management and Emergency Response". http://www.un-spider.org/space-application-matrix (Last accessed on 2018, Feb. 12).

Voigt, S., Giulio-Tonolo, F., Lyons, J., Kučera, J., Jones, B., Schneiderhan, T., Platzeck, G., Kaku, K., Hazarika, M.K., Czaran, L. and Li, S., 2016. Global trends in satellite-based emergency mapping. In: Science, 353(6296), pp.247-252.

Williams, J.G., Rosser, N.J., Kincey, M.E., Benjamin, J., Oven, K.J., Densmore, A.L., Milledge, D.G., Robinson, T.R., Jordan, C.A. and Dijkstra, T.A., 2018. Satellite-based emergency mapping using optical imagery: experience and reflections from the 2015 Nepal earthquakes. In: Natural Hazards and Earth System Sciences, 18(1), pp.185-205. 